\title{
Modelos de regressão aleatória para avaliação da curva de crescimento em matrizes de codorna de corte
}

\author{
Random regression models for growth evaluation of meat-type quail hens
}

\author{
Bruno Bastos Teixeira' ${ }^{\text {* }}$ Ricardo Frederico Euclydes ${ }^{\mathrm{I}}$ Rafael Bastos Teixeira ${ }^{\mathrm{II}}$ \\ Luciano Pinheiro da Silva ${ }^{I}$ Robledo de Almeida Torres ${ }^{I}$ Helmut Gonçalves Lehner ${ }^{I}$ \\ Giovani da Costa Caetano ${ }^{\mathrm{I}}$ Aline Campores Crispim ${ }^{\mathrm{I}}$
}

\section{RESUMO}

Objetivou-se comparar diferentes modelos de regressão aleatória por meio de funções polinomiais de Legendre de diferentes ordens, para avaliar o que melhor se ajusta ao estudo genético da curva de crescimento de codornas de corte. Foram avaliados dados de 2136 matrizes de codorna de corte, dos quais 1026 pertenciam ao grupo genético UFV1 e 1110 ao grupo UFV2. As codornas foram pesadas nos 1으, 7으, $14^{\circ}, 21^{\circ}, 28^{\circ}, 35^{\circ}, 42^{\circ}, 77^{\circ}, 112^{\circ}$ e $147^{\circ}$ dias de idade e seus pesos utilizados para a análise. Foram testadas duas possíveis modelagens de variância residual heterogênea, sendo agrupadas em 3 e 5 classes de idade. Após, foi realizado o estudo do modelo de regressão aleatória que melhor aplica-se à curva de crescimento das codornas. A comparação entre os modelos foi feita pelo Critério de Informação de Akaike (AIC), Critério de Informação Bayesiano de Schwarz (BIC), Logaritmo da função de verossimilhança $\left(\log _{e} L\right)$ e teste da razão de verossimilhança (LRT), ao nível de $1 \%$. O modelo que considerou a heterogeneidade de variância residual CL3 mostrou-se adequado à linhagem UFV1, e o modelo CL5 à linhagem UFV2. Uma função polinomial de Legendre com ordem 5, para efeito genético aditivo direto e 5 para efeito permanente de animal, para a linhagem UFV1 e, com ordem 3, para efeito genético aditivo direto e 5 para efeito permanente de animal para a linhagem UFV2, deve ser utilizada na avaliação genética da curva de crescimento das codornas de corte.

Palavras-chave: Coturnix coturnix, herdabilidade, heterogeneidade de variância, polinômio de Legendre.

\section{ABSTRACT}

The objective was to compare different random regression models using Legendre polynomial functions of different orders, to evaluate what best fits the genetic study of the growth curve of meat quails. It was evaluated data from 2136 cut dies quail, of which 1026 belonged to genetic group UFV1 and 1110 the group UFV2. Quail were weighed at 10, $70,140,210,280,350,420,770,1120$ and 1470 days of age, and weights used for the analysis. It was tested two possible modeling of heterogeneous residual variance, which was grouped into three five age classes. After, it was studied the random regression mode, which is better applied to the growth curve quail. The comparison between models was made by the Akaike Information Criterion (AIC), Bayesian Information Criterion of Schwarz (BIC), logarithm of the likelihood function (Loge L) and the likelihood ratio test (LRT), at 1\%. The model which considered the heterogeneity of residual variance CL3 was adequate to UFV1 lineage and the lineage model CL5 UFV2. A Legendre polynomial with order 5, for direct genetic effect and 5 for the permanent animal to UFV1 bloodline and order 3 for direct genetic effect and 5 for the permanent animal for UFV2 bloodline should be used in genetic evaluation of the growth curve of meat quails.

Key words: Coturnix coturnix, heritability, heterogeneous variance, Legendre polynomial.

\section{INTRODUÇÃO}

A avaliação da curva de crescimento das matrizes de codorna de corte, ao longo do ciclo da produção de ovos, é de grande importância, visto que o peso corporal das codornas influencia diversas características de produção e reprodução. O crescimento inicial, pode, de outra forma, aumentar a

\footnotetext{
IDepartamento de Zootecnia, Universidade Federal de Viçosa (UFV), 36571-000, Viçosa, MG, Brasil. E-mail: brunobatzootecnia@yahoo.com.br.*Autor para correspondência.

IIDepartamento de Zootecnia, Instituto Federal Minas Gerais, Campus Bambuí, Bambuí, MG, Brasil.
} 
deposição de gordura que tem efeito prejudicial nas características reprodutivas.

Os ciclos envolvidos na produção de linhagens, especialmente o referente à seleção das linhas puras, devem ser embasados nas variações genéticas das características. Para a seleção das linhagens, pode-se optar pelo uso de modelos de regressão aleatória (MEYER, 2000; AKBAS et al., 2004), que são uma alternativa para modelar as características de crescimento, por serem medidas repetidas ao longo da vida do animal.

Segundo BONAFÉ et al. (2011a), modelos de regressão aleatória permitem a obtenção de diferentes curvas de valores genéticos associadas aos diferentes indivíduos e consideram as mudanças nas variâncias genéticas e residuais, ao longo do tempo.

Ao ajustar um modelo de regressão aleatória, implicitamente, ajusta-se uma função contínua, que permite descrever as mudanças genéticas e ambientais, em função do tempo. Os polinômios ortogonais de Legendre podem requerer ordens de ajustes diferentes para cada efeito aleatório contido no modelo de análise. A falta de um modelo apropriado, isto é, o desconhecimento da ordem de ajuste mais apropriada para a função empregada, pode influenciar erroneamente a partição da variância fenotípica, nas variâncias atribuídas aos efeitos incluídos no modelo (SARMENTO et al., 2010).

Dessa forma, objetivou-se com este trabalho comparar diferentes modelos de regressão aleatória por meio de funções polinomiais de Legendre de diferentes ordens, para avaliar o que melhor se ajusta ao estudo genético da curva de crescimento de codornas de corte.

\section{MATERIAL E MÉTODOS}

Foram avaliados dados de 2136 matrizes de codorna de corte, dos quais 1026 codornas pertenciam ao grupo genético UFV1 e 1110 ao grupo genético UFV2. As codornas foram pesadas nos $1^{\circ}$, 7o, $14^{\circ}, 2^{\circ}$,

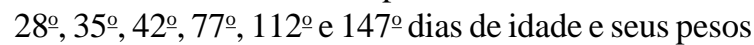
foram utilizados para a análise.

Os pesos corporais foram analisados por meio de modelo animal em regressão aleatória. As regressões fixas foram representadas por funções contínuas, cujas idades foram descritas em termos de polinômios ortogonais de Legendre, de ordem três (quadrático) e as regressões aleatórias genética aditiva e de ambiente permanente com ordem variando de 1 a 5. O modelo pode ser representado como segue:

$y_{i j}=F+\sum_{m=1}^{K} \beta_{m} \phi_{m}+\sum_{m=1}^{K a} \alpha_{i m} \phi_{m}+\sum_{m=1}^{K c} \rho_{i m} \phi_{m}+\varepsilon_{i j}$
Em que $y_{i j}$ é o peso no dia $j$, da codorna $i ; F$ refere-se aos efeitos fixos, constituídos pelo grupo de contemporâneos; $\beta_{m}$ é o coeficiente de regressão fixo do peso sobre o polinômio de Legendre $m$, representado por uma função quadrática, para modelar a curva média de crescimento da população; $\alpha_{i m}$ e $\rho_{i m}$ são os coeficientes de regressão genético-aditivo direto e de ambiente permanente do animal, respectivamente, para a codorna $i$; $k$ é a ordem de ajuste do polinômio de Legendre para o efeito fixo; $k_{a}$ e $k_{c}$ são as ordens de ajuste dos polinômios de Legendre, para cada efeito aleatório; $\phi_{m}$ é a função polinomial de Legendre $m$ da idade padronizada $(-1<$ idade $<1)$; e $\varepsilon_{i j}$ denota o efeito aleatório residual.

Foi considerada distribuição normal dos resíduos e as medidas de erros assumidas como independentes.

O modelo geral indicado anteriormente, com suas pressuposições, pode ser descrito na forma matricial da seguinte maneira: $y=X b+Z_{1} a+Z_{2} c+e$

$$
E\left[\begin{array}{c}
y \\
a \\
c \\
e
\end{array}\right]=\left[\begin{array}{c}
X b \\
0 \\
0 \\
0
\end{array}\right] \quad \text { e } \begin{gathered}
\operatorname{Var}(c)=K_{c} \otimes I_{N_{d}}, \\
\operatorname{Var}(e)=R
\end{gathered}
$$

Em que, $y$ é o vetor de observações referentes a $N_{d}$ codornas; $b$ é o vetor que contém os efeitos fixos e os coeficientes $b_{m}$ da regressão fixa; $a$ é o vetor $k_{a} \times N$ de coeficientes de regressão aleatória genético-aditivos diretos, em que $N$ denota o número total de codornas na matriz dos numeradores do coeficiente de parentesco entre os indivíduos; $c$ é o vetor $k_{c} \times N_{d}$ de coeficientes de regressão aleatória de ambiente permanente de animal; $e$ é o vetor de erros aleatórios; $X$ refere-se à matriz de incidência dos coeficientes de regressão fixos e efeitos fixos; $Z_{1}$ e $Z_{2}$ refere-se às matrizes de incidência dos coeficientes de regressão aleatória genético-aditivos direto e ambiente permanente de animal, respectivamente; $k_{a}$ e $k_{c}$ são matrizes de (co)variâncias entre os coeficientes de regressão aleatórios genético aditivo direto e ambiente permanente de animal, respectivamente; A é a matriz dos numeradores do coeficiente de parentesco entre os indivíduos; $I_{N d}$ é a matriz identidade de dimensão $N_{\mathrm{d}}$; $\otimes$ é o operador produto direto; $R$ é a matriz diagonal de variâncias residuais, ou seja, $R=I \sigma_{e}^{2}$.

Foram testadas duas possíveis modelagens de variância residual heterogênea, sendo agrupadas em 3 classes de idade: CL3: 1-7-14, 21-28-35 dias e 4277-112-147 dias e 5 classes de idade: CL5: 1-7, 14-21, 28-35, 42-77 e 112-147 dias de idade. 
Após a escolha do número de classes de variância residual a ser utilizada na análise das matrizes das linhagens UFV1 e UFV2, foi realizado o estudo do modelo de regressão aleatória que melhor se aplica à curva de crescimento das matrizes de codornas de corte.

As (co)variâncias dos coeficientes de regressão aleatória genéticos aditivos e ambiente permanente de animal, conforme o modelo ajustado, foram estimadas pelo método da máxima verossimilhança restrita (REML), usando o programa software DFREML (Derivative-Free Restricted Maximum Likelihood), através do subprograma DXMRR (Model Random Regression), descrito por MEYER (1998), que permite a estimação de componentes de (co)variância em modelos de regressão aleatória.

Os efeitos aleatórios, como proporção da variância fenotípica, para cada peso, no dia t da

pesagem, foi obtido como segue: $\hat{h}_{t_{i}}^{2}=\frac{\hat{\sigma}_{a t_{i}}^{2}}{\hat{\sigma}_{a_{i}}^{2}+\hat{\sigma}_{c t_{i}}^{2}+\hat{\sigma}_{e_{i}}^{2}}$; $\hat{c}_{t_{i}}^{2}=\frac{\hat{\sigma}_{c t_{i}}^{2}}{\hat{\sigma}_{a t_{i}}^{2}+\hat{\sigma}_{c t_{i}}^{2}+\hat{\sigma}_{e_{i}}^{2}} \quad$ em que, $\hat{h}_{t_{i}}^{2}$ é a estimativa da herdabilidade para cada peso, no dia t da pesagem; $\hat{c}_{t_{i}}^{2}$ é a proporção da variância de ambiente permanente em relação à fenotípica, no dia t da pesagem; $\hat{\sigma}_{a t_{i}}^{2}$ a estimativa da variância genética aditiva para cada peso, no dia $t$ da pesagem; $\hat{\sigma}_{c t}^{2}$ é a estimativa da variância de ambiente permanente, para cada peso, no dia t da pesagem; $\hat{\sigma}_{e_{i}}^{2}$ é a estimativa da variância residual.

As correlações genéticas e de ambiente permanente, entre os pesos nos diferentes dias de pesagem, $t_{i}$ e $t_{j}$, foram estimadas por:

$r_{a t_{i} t_{j}}=\frac{\hat{\sigma}_{a t_{i} t_{j}}}{\sqrt{\hat{\sigma}_{a t_{i}}^{2} \times \hat{\sigma}_{a t_{j}}^{2}}} \mathrm{e} r_{c t_{i} t_{j}}=\frac{\hat{\sigma}_{c t_{i} t_{j}}}{\sqrt{\hat{\sigma}_{c t_{i}}^{2} \times \hat{\sigma}_{c t_{j}}^{2}}}$, em que

$r_{a_{i} t_{j}}$ é a correlação genética entre os pesos, nos diferentes dias de pesagem, $t_{i}$ e $t_{j} ; r_{c_{i} t_{j}}$ é a correlação de ambiente permanente entre os pesos, nos diferentes dias de pesagem, $t_{i}$ e $t_{j} ; \hat{\sigma}_{a_{t} t_{i}}$ é a covariância genética aditiva entre os pesos, nos diferentes dias de pesagem, $t_{i}$ e $t_{j}$; $\hat{\sigma}_{c t, t i}$ é a covariância de ambiente permanente entre os pesos, nos diferentes dias de pesagem, $t_{i}$ e $t_{j} ; \quad \hat{\sigma}_{a t_{i}}^{2} \mathrm{e}$ $\hat{\sigma}_{a t_{j}}^{2}$ são as variâncias genética aditiva dos pesos, nos diferentes dias de pesagem $t_{i}$ e $t_{j}$; $\hat{\sigma}_{c t_{i}}^{2}$ e $\hat{\sigma}_{c t_{j}}^{2}$ são as variâncias de ambiente permanente dos pesos, nos diferentes dias de pesagem $t_{i}$ e $t_{j}$.

A comparação entre os modelos foi feita pelo Critério de Informação de Akaike (AIC), Critério de Informação Bayesiano de Schwarz (BIC), Logaritmo da função de verossimilhança $\left(\log _{e} L\right)$ e teste da razão de verossimilhança (LRT), ao nível de $1 \%$ de probabilidade, com distribuição $\chi^{2}$, para testar diferenças entre os modelos estudados. Esses critérios são baseados no logaritmo da função de verossimilhança e se diferem, basicamente, pela importância dada ao número de parâmetros no modelo.

Os critérios AIC e BIC foram obtidos por: $A I C=-2 \log _{e} L+2 p$ e $B I C=-2 \log _{e} L+2 p \log _{e}(N-r)$, em que p se refere ao número de parâmetros do modelo, $\mathrm{N}$ é o número total de observações e $\mathrm{r}$ é o posto da matriz de incidência para os efeitos fixos. Menores valores de AIC e BIC emaiores valores de $\log _{e}$ Lindicam modelos mais adequados (BURNHAM \& ANDERSON, 2004). A estatística LRT foi obtida pela seguinte expressão: $L R T_{i j}=2 \log _{e} L_{i}-2 \log _{e} L_{j}$, em que $\log _{\mathrm{e}} L_{\mathrm{i}}$ é o máximo da função de verossimilhança para o modelo completo i e $\log _{e} L_{j}$ o máximo da função de verossimilhança para o modelo reduzido j. A estimativa LRT foi comparada com o valor da distribuição quiquadrado, com d graus de liberdade e nível de significância de $1 \%$, sendo d a diferença entre o número de parâmetros estimados pelos modelos completo e reduzido (aninhados). As conclusões foram feitas da seguinte maneira: se $L R T>\chi^{2}(0,01 ; d)$ o teste seria significativo e o modelo completo forneceria melhor ajuste em relação ao modelo reduzido. A hipótese de nulidade testada foi de que os modelos, completo e reduzido, não diferiram entre si.

\section{RESULTADOS E DISCUSSÃO}

Seguem, na tabela 1, os resultados AIC, BIC, $2 \log _{e} L$, NP (número de parâmetros) e LRT, obtidos para as diferentes classes de heterogeneidade. De acordo com todos os critérios utilizados para avaliar a qualidade de ajuste, o modelo que considerou a heterogeneidade de variância residual CL3 mostrou-se adequado à linhagem UFV1, e o modelo CL5 mostrouse um modelo adequado à linhagem UFV2.

Para a linhagem UFV1, não houve aumentos significativos $(\mathrm{P}<0,01)$ no $\log _{\mathrm{e}} \mathrm{L}$, pelo teste da razão de verossimilhança (LRT) com o incremento do número de classes heterogêneas. Não se observam mudanças consideráveis nos valores de AIC e BIC, sendo esses fatores considerados na avaliação dos modelos. O modelo CL3, com três classes, poderia ser utilizado sem perdas na qualidade do ajuste de variância dos dados para a linhagem UFV1, além de ser mais parcimonioso em relação ao modelo CL5.

SARMENTO et al. (2010), ao trabalharem com a curva de crescimento de ovinos, verificou que o modelo, considerando homogeneidade de variâncias residuais, mostrou-se inadequado, sendo que a 
Tabela 1 - Logaritmo da função de verossimilhança (2 $\left.\log _{e} \mathrm{~L}\right)$, critério de informação de Akaike (AIC), critério de informação Bayesiano (BIC), número de parâmetros (NP) e Logaritmo da função de máxima verossimilhança (LRT), estimados para as modelagens CL3 e CL5 de variância residual heterogênea, para as linhagens UFV1 e UFV2.

\begin{tabular}{|c|c|c|c|c|c|c|}
\hline Linhagens & Modelo & $2 \log _{e} L$ & AIC & $\mathrm{BIC}$ & NP & LRT \\
\hline \multirow{2}{*}{ UFV1 } & CL3 & $-42.317,174$ & 84668.34846 & 84792.66618 & 15 & - \\
\hline & CL5 & $-42.307,945$ & 84645.89165 & 84755.58375 & 17 & $-9.2284^{\mathrm{NS}}$ \\
\hline \multirow{2}{*}{ UFV2 } & CL3 & $-40.155,719$ & 80341.43923 & 80449.9735 & 15 & - \\
\hline & CL5 & $-39.836,152$ & 79706.30415 & 79829.30966 & 17 & $-319.821^{* *}$ \\
\hline VEM & \multicolumn{5}{|c|}{--------------------------------------------------------------Linhagem } & 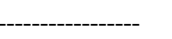 \\
\hline Modelo $^{1}$ & $2 \log _{\mathrm{e}} \mathrm{L}$ & AIC & \multicolumn{2}{|c|}{ BIC } & NP & LRT \\
\hline 33 & $-42.307,945$ & 84.645 .891 & \multicolumn{2}{|c|}{$84.755,583$} & 15 & \\
\hline 34 & $-42.307,945$ & 84.246 .109 & \multicolumn{2}{|c|}{$84.385,052$} & 19 & $0,00001^{\mathrm{NS}}$ \\
\hline 35 & $-40.032,233$ & 80.112 .467 & \multicolumn{2}{|c|}{$80.287,974$} & 24 & $2.275,717^{* *}$ \\
\hline 44 & $-41.590,122$ & 83.226 .244 & \multicolumn{2}{|c|}{$83.394,438$} & 23 & $1.557,881^{* *}$ \\
\hline 45 & $-40.015,913$ & 80.087 .826 & \multicolumn{2}{|c|}{$80.292,585$} & 28 & $1.554,208 * *$ \\
\hline 55 & $-39.376,798$ & 78.819 .596 & \multicolumn{2}{|c|}{$79.060,919$} & 33 & $639,115^{* *}$ \\
\hline \multicolumn{7}{|l|}{ VEM } \\
\hline Modelo $^{1}$ & $2 \log _{e} L$ & \multicolumn{3}{|l|}{ AIC } & NP & LRT \\
\hline 33 & $-39.836,152$ & \multicolumn{3}{|c|}{$79.829,308$} & 17 & \\
\hline 34 & $-43.329,051$ & $86.700,103$ & \multicolumn{2}{|c|}{$86.852,051$} & 21 & $3.492,898^{* *}$ \\
\hline 35 & $-38.221,809$ & 76.495,618 & \multicolumn{2}{|c|}{$76.683,744$} & 26 & $5.017,962 * *$ \\
\hline 44 & $-38.576,053$ & $77.202,106$ & 77 & 997 & 25 & $354,244^{* *}$ \\
\hline 45 & $-39.181,735$ & $78.423,470$ & 78. & 538 & 30 & $605,6818^{* *}$ \\
\hline 55 & $-38.501,275$ & $77.072,550$ & 77. & 796 & 35 & $680,4601^{* *}$ \\
\hline
\end{tabular}

** significativo a $1 \%$ de probabilidade; NS = Não Significativo; ${ }^{\mathbf{1}}$ ordem de ajuste para os efeitos genéticos aditivo direto e ambiente permanente; $\mathrm{CL}$ = classes de idades; VEM = Valores estimados para os modelos estudados nas linhagens de codorna de corte.

variância residual, contendo sete classes heterogêneas, proporcionou melhor ajuste, embora um mais parcimonioso, com cinco classes, poderia ser utilizado, sem perdas na qualidade de ajuste da variância nos dados.

BONAFÉ et al. (2011a), ao estudarem a curva de crescimento de codornas, mostra que as estimativas de variâncias, herdabilidades e correlações foram influenciadas pela modelagem da variância residual. Faz-se necessária a utilização de heterogeneidade de variância, para modelar as variâncias associadas à curva de crescimento de codornas de corte.

Também, na tabela 1, estão apresentados os valores de $2 \log _{\mathrm{e}} \mathrm{L}$, AIC, BIC, NP e LRT para os valores estimados para os modelos estudados nas linhagens de codornas de corte. De acordo com $2 \mathrm{Log}_{\mathrm{e}}$ $\mathrm{L}$, as ordens mais altas dos polinômios para os efeitos produziram maiores valores, o que sugere que o modelo mais parametrizado (modelo 55, com 33 parâmetros a serem estimados), com ordem 5 para os efeitos genético aditivo direto e 5 para ambiente permanente de animal, proporcionou melhor ajuste para a linhagens UFV1 e o modelo 35 (com 26 parâmetros a serem estimados) com ordem 3 para os efeitos genético aditivo direto e ordem
5 para ambiente permanente de animal, proporcionou melhor ajuste para a UFV2.

$\mathrm{Na}$ tabela 2, estão apresentadas as estimativas de variância genética aditiva e variâncias de ambiente permanente, para os modelos estudados nas linhagens UFV1 e UFV2. A variância genética aditiva estimada para as codornas da linhagem UFV1 foi semelhante até os 28 dias de idade, apresentando maior variação para o modelo 55, a partir dessa idade. Na linhagem UFV2, observou-se uma maior oscilação entre as variâncias aditivas nos diversos modelos estudados. BONAFÉ et al. (2011a), ao analisarem a variância genética aditiva em codornas de corte, obtiveram estimativas semelhantes, porém encontraram maior variação, a partir dos 21 dias de idade.

Observou-se queda nas estimativas de variância genética aditiva a partir dos 112 dias de idade para todos os modelos estudados na linhagem UFV1. Na linhagem UFV2, observou-se esta queda, exceto para os modelos 45 e 55. Estimativas de variâncias genéticas aditivas diretas crescentes, ao longo da trajetória, foram relatadas em ovinos de corte (SARMENTO et al., 2010) e em bovinos de corte (ALBUQUERQUE \& MEYER, 2001), que também

Ciência Rural, v.42, n.9, set, 2012. 
Tabela 2 - Estimativas de variância genética aditiva (VGA) e de variâncias de ambiente permanente (VAP) de peso por idade, para as linhagens de codornas de corte UFV1 e UFV2.

\begin{tabular}{|c|c|c|c|c|c|c|c|c|c|c|}
\hline \multirow{2}{*}{$\begin{array}{l}\text { VGA } \\
\text { Modelo }\end{array}$} & \multicolumn{10}{|c|}{ Linhagem UFV1 } \\
\hline & 1 & 7 & 14 & 21 & 28 & 35 & 42 & 77 & 112 & 147 \\
\hline 33 & 13,48 & 1,88 & 45,71 & 134,9 & 254,2 & 389,5 & 528,86 & 980,48 & 692,65 & 64,28 \\
\hline 34 & 16,74 & 0,5 & 34,3 & 111,4 & 218,8 & 345,1 & 476,66 & $1.003,5$ & 911,88 & 341,2 \\
\hline 35 & 0,73 & 11,99 & 66,92 & 157,5 & 274,8 & 410,5 & 556,86 & $1.232,1$ & $1.441,9$ & 1.037, \\
\hline 44 & 44,78 & 1,5 & 55,72 & 131,4 & 206,8 & 308,2 & 484,49 & $3.598,7$ & $6.064,5$ & 451,2 \\
\hline 45 & 0,76 & 13,56 & 69,65 & 159,2 & 277,7 & 424,0 & 599,02 & $1.805,7$ & $2.446,5$ & 916,5 \\
\hline 55 & 8,71 & 114,2 & 247,7 & 220,4 & 272,3 & 617,5 & $1.265,2$ & $2.490,7$ & $6.451,6$ & $3.14,1$ \\
\hline \multicolumn{11}{|l|}{ VGA } \\
\hline Modelo & 1 & 7 & 14 & 21 & 28 & 35 & 42 & 77 & 112 & 147 \\
\hline 33 & 76,73 & 32,15 & 51,01 & 127,7 & 244,6 & 386,2 & 538,53 & $1.111,5$ & 973,43 & 529,3 \\
\hline 34 & 0,11 & 2,2 & 9,85 & 21,27 & 34,81 & 49,07 & 62,82 & 95,42 & 49,11 & 0,15 \\
\hline 35 & 2,22 & 8,51 & 59,23 & 146,0 & 258,6 & 387,6 & 524,73 & $1.103,2$ & $1.165,4$ & 721,2 \\
\hline 44 & 57,09 & 1,11 & 80,11 & 219,6 & 358,6 & 466,8 & 535,72 & 670,04 & $1.106,6$ & 692,2 \\
\hline 45 & 712,9 & 106,1 & 32,94 & 301,9 & 671,6 & 996,1 & $1.202,7$ & 684,7 & 274,14 & 437,5 \\
\hline 55 & 29,5 & 21,57 & 36,91 & 94,45 & 203,3 & 348,9 & 495,08 & 230,36 & 421,0 & 889,8 \\
\hline \multicolumn{11}{|l|}{ VAP } \\
\hline Modelo & 1 & 7 & 14 & 21 & 28 & 35 & 42 & 77 & 112 & 147 \\
\hline 33 & 0,14 & 0,24 & 2,05 & 5,19 & 9,19 & 13,65 & 18,18 & 32,94 & 24,22 & 3,3 \\
\hline 34 & 69,75 & 0,66 & 44,23 & 64,78 & 33,15 & 7,86 & 89,0 & $4.466,9$ & $8.401,4$ & 28,05 \\
\hline 35 & 14,73 & 178,3 & 273,3 & 63,25 & 126,0 & 899,5 & $2.331,9$ & $2.767,1$ & $6.292,5$ & 9.468, \\
\hline 44 & 0,57 & 0,32 & 3,64 & 9,17 & 15,61 & 22,01 & 27,79 & 40,56 & 37,49 & 46,1 \\
\hline 45 & 17,84 & 183,6 & 239,8 & 98,73 & 54,1 & 603,2 & $1.727,2$ & $1.600,4$ & $8.324,7$ & 3.153, \\
\hline 55 & 0,36 & 4,45 & 14,34 & 20,63 & 20,56 & 15,32 & 8,03 & 28,66 & 92,37 & 10,5 \\
\hline \multicolumn{11}{|l|}{ VAP } \\
\hline Modelo & 1 & 7 & 14 & 21 & 28 & 35 & 42 & 77 & 112 & 147 \\
\hline 33 & 2,91 & 0,41 & 2,07 & 8,79 & 19,11 & 32,03 & 46,62 & 118,55 & 143,4 & 101,8 \\
\hline 34 & 25,63 & 146,6 & 400,0 & 427,3 & 336,6 & 509,3 & $1.404,3$ & 22.682, & 27.894, & 9.423, \\
\hline 35 & 1,44 & 13,83 & 34,56 & 225,2 & 795,5 & 1.778, & $2.991,9$ & $3.242,5$ & $2.584,5$ & 1.648, \\
\hline 44 & 1,34 & 0,11 & 2,31 & 10,86 & 27,51 & 53,68 & 90,17 & 404,07 & 689,34 & 502,9 \\
\hline 45 & 727,8 & 189,3 & 70,69 & 80,89 & 111,2 & 189,5 & 358,1 & $1.561,3$ & $1.203,9$ & 1.151, \\
\hline 55 & 11,93 & 24,62 & 81,89 & 110,1 & 172,6 & 352,6 & 699,7 & $4.932,4$ & 10.723, & 142,4 \\
\hline
\end{tabular}

descreveram estimativas crescentes ao longo do período estudado, com ligeira tendência de diminuição, ao final da curva de crescimento.

Resultados semelhantes também foram apresentados por DIONELLO et al., 2008, que, em trabalho com codornas europeias, admitindo Polinômio de Legendre de ordem três, encontraram variância genética aditiva, que variou de 1,18 a 532,8, com oscilações associadas ao avançar da idade.

Uma possível explicação para a trajetória da variância fenotípica são os efeitos provocados por uma mudança de escala, sendo que populações que apresentam um peso médio mais alto têm uma variância associada maior e, a partir de certa idade, com a estabilização do crescimento, essa variância se reduz. O mesmo pode ser observado com relação à variância genética aditiva, que é proporcional à fenotípica.
As estimativas de variância de ambiente permanente foram erráticas para as linhagens UFV1 e UFV2, ou seja, apresentaram oscilações até 147 dias de idade, porém com tendências de aumento com a idade. BONAFÉ et al. (2011a) encontraram estimativas de efeito de ambiente permanente crescentes, do nascimento aos 42 dias de idade. AKBAS et al. (2004) e WINTER et al. (2006) encontraram valores de covariâncias para ambiente permanente, positivas e crescentes, entre os pesos corporais em codornas de corte.

$\mathrm{Na}$ tabela 3, estão apresentadas as estimativas de variâncias fenotípicas, variâncias residuais e herdabilidades para os modelos estudados nas linhagens UFV1 e UFV2. Assim como encontrados por AKBAS et al. (2004) e BONAFÉ et al. (2011a), todos os componentes de variância aumentaram com a idade 
Tabela 3 - Estimativas de variâncias fenotípicas (VF), variâncias residuais (VR), herdabilidades $\left(\mathrm{h}^{2}\right)$ de peso por idade, para as linhagens de codornas de corte UFV1 e UFV2.

\begin{tabular}{|c|c|c|c|c|c|c|c|c|c|c|}
\hline \multirow{2}{*}{$\begin{array}{l}\text { VF } \\
\text { Modelo }\end{array}$} & \multicolumn{10}{|c|}{ Linhagem UFV1 } \\
\hline & 1 & 7 & 14 & 21 & 28 & 35 & 42 & 77 & 112 & 147 \\
\hline 33 & 331,5 & 320,0 & 365,6 & 260,8 & 384,0 & 523,8 & 5.757, & 6.223, & 5.927, & $5.277,9$ \\
\hline 34 & 546,0 & 460,6 & 538,0 & 338,7 & 414,6 & 515,6 & 1.914, & 6.815, & 10.658 & $1.714,6$ \\
\hline 35 & 43,97 & 218,8 & 368,8 & 395,5 & 575,6 & 1.484 & 5.984, & 7.094, & 10.829 & 13.601, \\
\hline 44 & 531,5 & 487,9 & 545,5 & 303,2 & 385,1 & 492,8 & 1.665, & 4.792, & 7.254 & $1.650,2$ \\
\hline 45 & 48,95 & 227,5 & 413,9 & 435,6 & 509,4 & 1.204 & 5.254 & 6.334, & 13.699 & $6.997,6$ \\
\hline 55 & 36,89 & 146,8 & 290,2 & 412,6 & 464,4 & 804,4 & 4.320 & 5.566 & 9.591, & $6.242,0$ \\
\hline \multicolumn{11}{|l|}{ VF } \\
\hline Modelo & 1 & 7 & 14 & 21 & 28 & 35 & 42 & 77 & 112 & 147 \\
\hline 33 & 310,3 & 262,8 & 290,6 & 374,1 & 1.739 & 1.894 & 3.436, & 4.081, & 3.792, & $3.306,5$ \\
\hline 34 & 482,5 & 606,0 & 1.195, & 1.233 & 371,4 & 558,4 & 13.528 & 34.838 & 47.273 & 28.753, \\
\hline 35 & 103,1 & 121,8 & 211,1 & 488,5 & 1.592 & 2.704 & 5.783, & 6.613, & 4.407, & $3.027,0$ \\
\hline 44 & 429,1 & 371,8 & 417,1 & 565,2 & 917,8 & 1.052 & 1.824, & 2.272 & 2.212 & $1.611,3$ \\
\hline 45 & 5.371, & 4.226 & 106,8 & 386,0 & 1.121 & 1.524 & 2.634, & 3.319, & 1.810 & $1.921,5$ \\
\hline 55 & 235,8 & 240,5 & 304,2 & 390,0 & 716,3 & 1.042 & 2.520 & 6.488 & 13.581 & $5.702,1$ \\
\hline \multicolumn{11}{|l|}{ VR } \\
\hline Modelo & 1 & 7 & 14 & 21 & 28 & 35 & 42 & 77 & 112 & 147 \\
\hline 33 & 317,9 & 317,9 & 317,9 & 120,6 & 120,6 & 120,6 & 5.120, & 5.120, & 5.120 & $5.120,3$ \\
\hline 34 & 459,50 & 459,5 & 459,5 & 162,6 & 162,6 & 162,6 & 1.345, & 1.345, & 1.345, & $1.345,0$ \\
\hline 35 & 28,501 & 28,50 & 28,50 & 174,7 & 174,7 & 174,7 & 3.095, & 3.095, & 3.095, & 3095,2 \\
\hline 44 & 486,16 & 486,1 & 486,1 & 162,6 & 162,6 & 162,6 & 1.152, & 1.152, & 1.152, & $1.152,8$ \\
\hline 45 & 29,842 & 29,84 & 29,84 & 173,0 & 173,0 & 173,8 & 2.977, & 2.977 & 2.977 & $2.977,1$ \\
\hline 55 & 28,135 & 28,13 & 28,13 & 171,5 & 171,5 & 171,5 & 3.047, & 3.047, & 3.047, & $3.047,3$ \\
\hline \multicolumn{11}{|l|}{ VR } \\
\hline Modelo & 1 & 7 & 14 & 21 & 28 & 35 & 42 & 77 & 112 & 147 \\
\hline 33 & 230,66 & 230,6 & 237,61 & 237,6 & 1.476 & 1.476 & 2.851, & 2.851, & 2.675 & $2.675,3$ \\
\hline 34 & 457,20 & 457,2 & 784,96 & 784,9 & 0,01 & 0,01 & 12.061 & 12.061 & 19.329 & 19.329, \\
\hline 35 & 99,466 & 99,46 & 117,32 & 117,3 & 538,4 & 538,4 & 2.267, & 2.267, & 657,64 & 657,64 \\
\hline 44 & 370,76 & 370,7 & 334,71 & 334,7 & 531,6 & 531,6 & 1.198, & 1.198, & 416,15 & 416,15 \\
\hline 45 & 3930,4 & 3930, & 3,159 & 3,159 & 338,9 & 338,9 & 1.073, & 1.073, & 332,64 & 332,64 \\
\hline 55 & 194,39 & 194,3 & 185,45 & 185,4 & 340,3 & 340,3 & 1.325 & 1.325, & 2.436 & $2.436,7$ \\
\hline \multicolumn{11}{|l|}{$h^{2}$} \\
\hline Modelo & 1 & 7 & 14 & 21 & 28 & 35 & 42 & 77 & 112 & 147 \\
\hline 33 & 0,04 & 0,05 & 0,12 & 0,51 & 0,66 & 0,74 & 0,09 & 0,15 & 0,11 & 0,12 \\
\hline 34 & 0,03 & 0,01 & 0,06 & 0,32 & 0,52 & 0,66 & 0,25 & 0,14 & 0,08 & 0,19 \\
\hline 35 & 0,01 & 0,05 & 0,18 & 0,39 & 0,47 & 0,27 & 0,09 & 0,17 & 0,13 & 0,07 \\
\hline 44 & 0,08 & 0,03 & 0,1 & 0,43 & 0,53 & 0,62 & 0,29 & 0,75 & 0,83 & 0,27 \\
\hline 45 & 0,01 & 0,05 & 0,16 & 0,36 & 0,54 & 0,35 & 0,11 & 0,28 & 0,17 & 0,13 \\
\hline 55 & 0,23 & 0,07 & 0,85 & 0,53 & 0,58 & 0,76 & 0,29 & 0,44 & 0,67 & 0,51 \\
\hline \multicolumn{11}{|l|}{$h^{2}$} \\
\hline Modelo & 1 & 7 & 14 & 21 & 28 & 35 & 42 & 77 & 112 & 147 \\
\hline 33 & 0,24 & 0,12 & 0,17 & 0,34 & 0,14 & 0,2 & 0,15 & 0,27 & 0,25 & 0,16 \\
\hline 34 & 0,03 & 0,03 & 0,08 & 0,17 & 0,09 & 0,08 & 0,46 & 0,27 & 0,1 & 0,05 \\
\hline 35 & 0,02 & 0,06 & 0,28 & 0,28 & 0,16 & 0,14 & 0,09 & 0,16 & 0,26 & 0,23 \\
\hline 44 & 0,13 & 0,02 & 0,19 & 0,38 & 0,39 & 0,44 & 0,29 & 0,29 & 0,5 & 0,42 \\
\hline 45 & 0,13 & 0,02 & 0,3 & 0,78 & 0,59 & 0,65 & 0,45 & 0,2 & 0,15 & 0,22 \\
\hline 55 & 0,12 & 0,08 & 0,12 & 0,24 & 0,28 & 0,33 & 0,19 & 0,35 & 0,3 & 0,15 \\
\hline
\end{tabular}

das codornas. A variância fenotípica estimada, para a linhagem UFV2, aumentou consideravelmente com o tempo, sendo observada queda, a partir do $77^{\circ}$ dia de idade.
As herdabilidades estimadas foram de médias a altas, exceto das características peso, aos 7 e 14 dias de idade. TON et al. (2006) encontraram herdabilidades baixas, que variaram de 0,05 a 0,07. Já 
DIONELLO et al. (2008) encontraram herdabilidade de 0,08 para peso ao nascimento, estando de acordo com o resultados encontrados.

DIONELLO et al. (2008), ao estudar o peso corporal do grupo genético EV1, obteve resultados semelhantes aos descritos por AKBAS et al. (2004) que, ao usarem modelos de regressão aleatória, para avaliarem o crescimento de codornas de corte, encontraram aumento linear nos valores de herdabilidades dos pesos corporais, até o 28을 dia de idade, com a variância genética dos pesos corporais avaliados reduzidos, ao final do período.

\section{CONCLUSÃO}

O modelo que considerou a heterogeneidade de variância residual CL3 mostrou-se adequado à linhagem UFV1, e o modelo CL5 à linhagem UFV2. Uma função polinomial de Legendre com ordem 5 , para efeito genético aditivo direto e 5 para efeito permanente de animal, para a linhagem UFV1 e com ordem 3, para efeito genético aditivo direto e 5 para efeito permanente de animal para a linhagem UFV2, deve ser utilizada na avaliação genética da curva de crescimento das codornas de corte em estudo.

\section{REFERÊNCIAS}

ALBURQUERQUE, L.G.; MEYER, K. Estimates of covariance functions for growth from birth to 630 days of age in Nelore catlle. Journal of animal science, v.79, p. 2776-2789, 2001. Disponível em: <http://jas.fass.org/cgi/reprint/79/11/ 2776>. Acesso em: 24 out. 2008.

AKBAS, Y. et al. Genetic parameters for quail body weights using a random regression model. South African Journal of Animal Science, v.34, n.2, p.104-109, 2004. Disponível em: <http://www.sasas.co.za/journal/1097>. Acesso em: 06 fev. 2008.
BONAFÉ, C.M. et al. Modelos de regressão aleatória para descrição da curva de crescimento de codornas de corte. Revista brasileira de zootecnia, v.40, n.4, p.765-771, 2011a. Disponível em: <http://dx.doi.org/10.1590/S1516-35982011000400009> Acesso em: 26 jun. 2011. doi: 10.1590/ S151635982011000400009 .

BURNHAM, K.P.; ANDERSON, D.R. Multimodel inference: understanding AIC and BIC in model selection. Sociological Methods and Researchers, v.33, n.2, p.261-304, 2004.

DIONELLO, N.J.L. et al. Estimativas da trajetória genética do crescimento de codornas de corte utilizando modelos de regressão aleatória. Arquivo Brasileiro de Medicina Veterinária e Zootecnia, v.60, n.6, p.454-460, 2008. Disponível em: <http://www.scielo.br/ scielo.php?script=sci_arttext\&pid=S010209352008000200027 > . Acesso em: 18 de out. 2009. doi: 10.1590/S010209352008000200027

MEYER, K. «DXMRR» - A program to estimate covariance functions for longitudinal data by REML. WORLD CONGRESS OF GENETICS APPLIED TO LIVESTOCK PRODUCTION, 6., 1998, Armidale. Anais... Armidale: University of New England, 1998. V.6. 120p. p.29.

MEYER, K. Random regressions to model phenotypic variation in monthly weights of Australian beef cows. Livestock Production Science, v.65, p.19-38, 2000. Disponível em: $<$ http://www.sciencedirect.com/science>. Acesso em: 10 de out. 2008. doi:10.1016/S0301-6226(99)00183-9.

SARMENTO, J.L.R. et al. Modelos de regressão aleatória na avaliação genética do crescimento de ovinos da raça Santa Inês. Revista Brasileira de Zootecnia, v.39, n.8, p.1723-1732, 2010.

TON, A.P.S. et al. Estimação de parâmetros genéticos e fenotípicos para o peso corporal em codornas de corte. In: REUNIÃO ANUAL DA SOCIEDADE BRASILEIRA DE ZOOTECNIA, 43., 2006, João Pessoa, PB. Anais... João Pessoa: SBZ, 2006. [V.43].

WINTER, E.M.W. Aplicação do método Bayesiano na estimação de correlações genéticas e fenotípicas de peso em codornas de corte em várias idades. Revista da Sociedade Brasileira de Zootecnia, v.35, p.1684-1690, 2006. 